\title{
WIMAX BASICS FROM PHY LAYER TO SCHEDUling AND MUlticASTing APPROACHES
}

\author{
Manal Al-bzoor ${ }^{1}$ and Khaled Elleithy ${ }^{2}$ \\ ${ }^{1}$ Department of Computer Science Engineering, University Of Connecticut, CT, USA \\ mbzoordengr. uconn. edu \\ ${ }^{2}$ Department of Computer Science Engineering, University Of Bridgeport, CT, USA \\ elleithyabridgeport.edu
}

\begin{abstract}
WiMAX (Worldwide Interoperability for Microwave Access) is an emerging broadband wireless technology for providing Last mile solutions for supporting higher bandwidth and multiple service classes with various quality of service requirement. The unique architecture of the WiMAX MAC and PHY layers that uses OFDMA to allocate multiple channels with different modulation schema and multiple time slots for each channel allows better adaptation of heterogeneous user's requirements. The main architecture in WiMAX uses PMP (Point to Multipoint), Mesh mode or the new MMR (Mobile Multi hop Mode) deployments where scheduling and multicasting have different approaches. In PMP SS (Subscriber Station) connects directly to BS (Base Station) in a single hop route so channel conditions adaptations and supporting QoS for classes of services is the key points in scheduling, admission control or multicasting, while in Mesh networks SS connects to other SS Stations or to the BS in a multi hop routes, the MMR mode extends the PMP mode in which the SS connects to either a relay station (RS) or to Bs. Both MMR and Mesh uses centralized or distributed scheduling with multicasting schemas based on scheduling trees for routing. In this paper a broad study is conducted About WiMAX technology PMP and Mesh deployments from main physical layers features with differentiation of MAC layer features to scheduling and multicasting approaches in both modes of operations.
\end{abstract}

\section{KEYWORDS}

WiMAX, PMP, Mesh, Scheduling WiMAX, and Multicasting WiMAX

\section{INTRODUCTION}

WiMAX is the end to end technology that provides low cost applications and last mile solution for broadband wireless access. WiMAX is based on the standard family defined by IEEE 802.16 which provides Coverage of up to 30 miles (last mile) compared to other technologies; DSL can cover 3 miles; WiFi can only cover 30 meters. WiMAX has unique characteristics which allows the base station to handle thousands of subscriber stations (SS), provides a collision- free MAC Uplink/downlink (UL/DL) channels, it also provides efficient handover procedure and Power control mechanism by introducing the sleeping mode for mobile stations. WiMAX Supports Data, Legacy voice systems, VoIP, TCP/IP, Applications with different QoS classes, and different level of guarantees. The basic two layers in IEEE 802.16 are the MAC and the physical (PHY) layers. The PHY layer Combines OFDM/OFDMA Orthogonal Frequency Division Multiplexing/ Multiple Access and uses multiple inputs multiple output antenna technology with an adaptive coding and Modulation schemas to support various bandwidth request demands issued by the MAC layers services. The MAC layer in turn provides a medium independent interface to the physical layer as the standard medium access functionality. 
International Journal of Computer Science \& Engineering Survey (IJCSES) Vol.2, No.1, Feb 2011

Even though WiMAX is a relatively new standard, there is a tremendous work conducted to address hot issues. But a very little work was conducted to classify and survey the work and mechanisms proposed for addressing design issues in WiMAX. Some Studies Focused in the cross layer design capabilities of PHY Layer and MAC Layer for a better Quality of Service. Other Studies proposed new Scheduling Techniques for WiMAX taking one or more of class of service type as a key design factor. A survey in [12] made a good effort in describing and classifying the scheduling algorithms proposed earlier for other technologies and the ones proposed specifically for WiMAX and are based on their use of the channel conditions. Only few studies was conducted to provide routing and multicasting schemas for WiMAX because in point to multipoint mode multicasting is defined by a specific Multicast Broadcast service while the mesh mode of WiMAX follows the same mechanisms and techniques proposed for earlier wireless mesh networks.

In This survey we provide a study of basic key design and research issues for WiMAX. We describe the WiMAX standard evolutions, Deployments, basic Protocol Layer Stack mainly the Physical and MAC Layers by addressing the characteristics and features in both Point to Point and Mesh deployments, we then give a description of the scheduling Mechanisms and studies proposed for both deployments and finally we give a brief description and differentiation of routing and multicasting schemas used with WiMAX.

\section{WiMAX Standards}

In 2001, the WiMAX forum introduced the first fixed Standard 802.16 with line of sight requirement using a single carrier frequency with $10-66 \mathrm{GHz}$ spectrum support. This basic standard provides theoretical rates of up to $134 \mathrm{Mbps}$. In January 2003, 802.16a standard was approved with a non line of site support of $2-11 \mathrm{GHz}$ frequency, where the first orthogonal frequency division Multiplexing (OFDM) and the mesh mode were added to WiMAX. The $802.16 \mathrm{~b} \& \mathrm{c}$ were amendment to the $802.16 \mathrm{a}$ and all of them were theoretical standards which later were grouped in the 2004 WiMAX standard 802.16d. The 802.16d known as the first fixed working standard of WiMAX supports data rates of up to 70Mbps, uses 256 point fast Fourier transform for Orthogonal Frequency Division Multiplexing (OFDM) and 2048 points transform OFDMA (OFD Multiple Access)[3][4]. In 2005 the mobile WiMAX IEEE 802.16e [5] was introduced with the following improvement over previous standards:

- It supports mobility by Introducing a Mobile Stations (MS) instead of SS. MS in this standard can stay connected during movement from one BS coverage area to another BS coverage area through efficient handover procedures between Base stations.

- It uses Scalable OFDMA (SOFDMA) technology to enhance spectrum efficiency and reduce cost in wide and narrow band channels. It obtains this scalability by allowing different FFT point values for each channel width to resulting in a constant carrier spacing.

- It adapts to the Advance antenna technology supporting the Multiple In Multiple Out MIMO technology and uses hybrid automatic repeat-request (HARQ) to enhance reliability

- Introduces Turbo Coding and Low-Density Parity Check (LDPC)

- It uses the downlink sub-channelization, allowing administrators to trade coverage for capacity or vice versa

In 2009 the WiMAX forum approved the 802.16j called the MMR mode that is just an extension to the original the PMP. MMR mode have a tree structure where intermediate subscriber stations can work as relays to forward traffic and is fully backward compatible with 802.16e standard. 


\section{WiMAX Deployments}

WiMAX architectures use either Point to Multipoint (PMP), Mesh, or Mobile Multihop Relay (MMR) mode. PMP architecture was introduced as the first standard of WiMAX in 2001. In this mode subscriber stations connects to the base station in a single hop route. In Mesh mode subscriber stations can communicate in an ad-hoc fashion. Mesh mode gained too much attention by researchers but was not much deployed in the real world because it supports only OFDM version and is not compatible with PMP with completely different frame structure and network entry procedure. The mobile multi hop relay (MMR) mode in $802.16 \mathrm{j}$ was introduced as an extension for PMP mode in IEEE 802.16e [6]. MMR outperforms PMP but its achieving higher throughput and enhancing coverage [6] Unlike PMP mode, MMR Supports both OFDM and OFDMA operations and is backward compatible wita h PMP mode. MMR differs from PMP by introducing the Relay Stations in which mobile station can use as an intermediate route forwarders to the BS in a like tree topology rather than a PMP or MESH topology. MMR consist of three network entities BS, relay station (RS) and mobile station (MS). RSs have the functionality of playing and intermediate forwarder to forwards traffic between any MS and the BS. RS was classified to work in two modes transparent or non transparent [6]. In transparent mode only data signals are forwarded and no control signals are allowed to pass. RS In non transparent is being also divided according to the scheduling role they play and follow as distributed or centralized. Distributed if they are allowed to share any scheduling decision and bandwidth allocation with the BS or centralized if RS are just forwarder and scheduling decisions are made by the BS. Radio links between entities in the MMR mode are named Access links (AL) if it connects MSs with RS and are called relay links (RL) if the radio link is between the RS and the BS [7]. Figure 1 shows an example of different WiMAX deployments in which BS can work as a gateway for the internet; MS is a mobile SS; RS is a SS that works as a relay agent to forward traffic flows to BSs or other RSs.

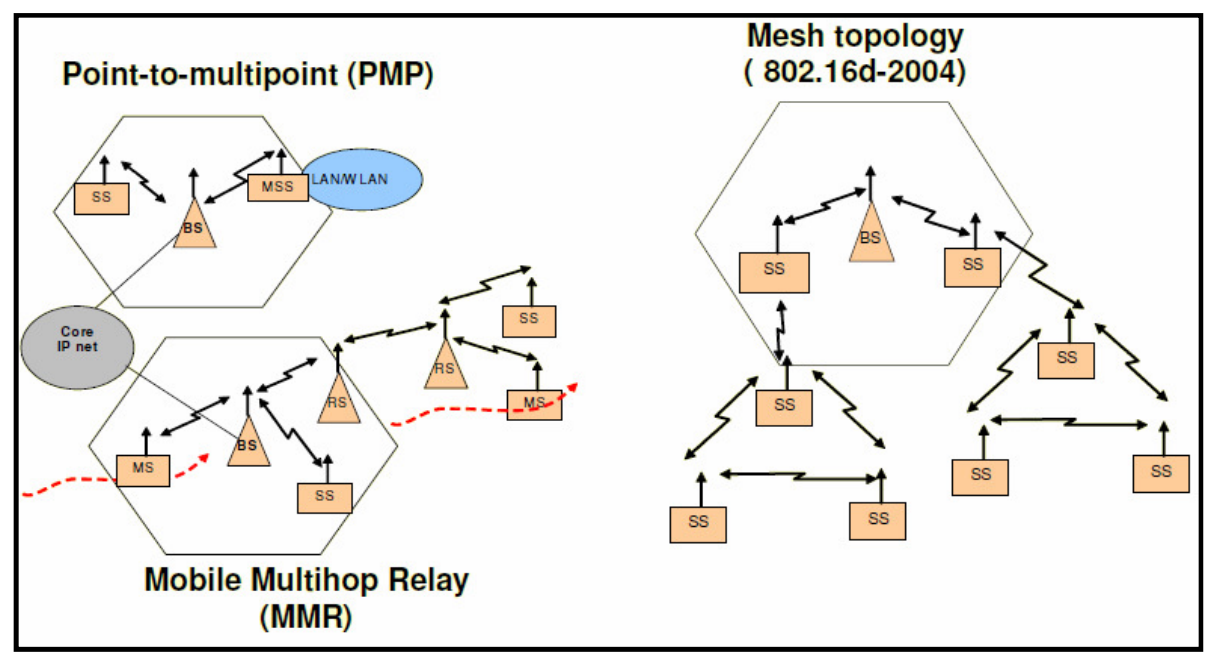

Figure 1.WiMAX Deployments

\section{Protocol Stack of WiMAX}

The two basic layers in WiMAX are the physical (PHY) layer and the Medium access control (MAC) layer and were designed mainly for the PMP mode. In This section we are introducing the main features in both layers. 
International Journal of Computer Science \& Engineering Survey (IJCSES) Vol.2, No.1, Feb 2011

\subsection{PHY LAYER}

The WiMAX physical layer (PHY) is designed to work with different specifications for licensed and unlicensed frequency bands. For example, one is based on a single carrier (SC) to support line of site with high data rates, others use orthogonal frequency division multiplexing (OFDM), and OFDMA to support both line of site(LOS) and none line of sight (NLOS) . [3][4][8][9]

4.1.1 OFDM /OFDMA: OFDM is an efficient modulation schema used for transmitting large amount of data over radio waves. OFDM is a multi carrier transmission method that is based on dividing the frequency of a carrier into orthogonal frequency sub carriers each carrying different stream of data and is can be modulated and coded separately. Since OFDM selects the sub carriers such that they are orthogonal to each other over the time duration, it limits or eliminates overlapping and the sub carrier interference. OFDMA is the access method that is based on the OFDM modulation technique to divide the carriers among the users to form sub channels. Each sub channel is allocated a separate coding and modulation parameters to allow are adapted separately, allowing channel optimization on a smaller scale (rather than using the same parameters for the whole channel). This technique optimizes the use of spectrum resources and enhances indoor coverage by assigning a robust scheme (yet, with low rates) to vulnerable links. OFDMA is an option in 802.16 (for fixed access), but it's not required for certifying 802.16 products. However, OFDMA is necessary in 802.16e devices and is required for certification. SOFDMA is an enhancement of OFDMA that scales the number of sub carriers in a channel using the four set of scaling factors $128,512,1,024$, and 2,048.

4.1.2. Modulation and Coding: WiMAX has the capability of changing its burst profile values depending on channel conditions per link and per connection. The two main parameters adjusted for the burst profile are the modulation and coding parameters used in addition shown in Table I below. For the Downlink burst profile other parameters are also specified like CINR threshold for entry and exit of this specific burst profile. In Uplink burst profile we can see a ranging data ratio. A Base station gets an estimate about the uplink channel conditions for each connection while it gets information about the downlink channel quality from control information provided by the mobile stations.

Table 1. Modulation and Coding used with Uplink and Downlink

\begin{tabular}{|c|c|c|}
\hline & Downlink & Uplink \\
\hline Modulation & $\begin{array}{lcc}\text { BPSK, QPSK, } & 16 \text { QAM, } & 64 \\
\text { QAM; BPSK } & \text { optional } & \text { for } \\
\text { OFDMA-PHY } & & \end{array}$ & $\begin{array}{l}\text { BPSK, QPSK, } 16 \text { QAM; } 64 \\
\text { QAM optional }\end{array}$ \\
\hline Coding & $\begin{array}{l}\text { Mandatory: convolutional codes } \\
\text { at rate } 1 / 2,2 / 3,3 / 4,5 / 6 \\
\text { Optional: convolutional turbo } \\
\text { codes at rate } 1 / 2,2 / 3,3 / 4,5 / 6 ; \\
\text { repetition codes at rate } 1 / 2,1 / 3 \text {, } \\
1 / 6 \text { LDPC, RS-Codes for } \\
\text { OFDM-PHY }\end{array}$ & 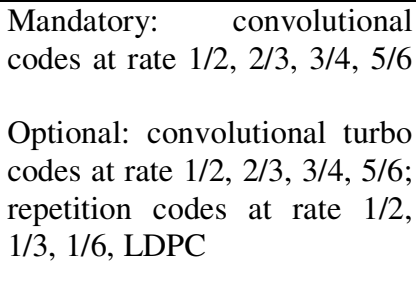 \\
\hline
\end{tabular}


4.1.3 PHY-Layer Data Rates: The design of physical layer in WiMAX allows multiple data rates that depends on parameters specified during the entry procedure and service request. The parameters that affects the physical layer data rate or what that is specified in the burst profile is determined from the modulation and coding schema used in addition to the communication channel bandwidth which makes the physical layer uniquely flexible. Table II provides an example of data rate obtained using different values for channel bandwidths, with various modulation and coding schemes.

Table 2. PHY Layer Data Rates

\begin{tabular}{|c|c|c|c|c|c|c|c|c|}
\hline Channel Bandunidth & \multicolumn{2}{|c|}{$3.5 \mathrm{MHz}$} & \multicolumn{2}{|c|}{$1.25 \mathrm{MHz}$} & \multicolumn{2}{|c|}{$5 \mathrm{MHz}$} & \multicolumn{2}{|c|}{$10 \mathrm{MHz}$} \\
\hline PHY mode & \multicolumn{2}{|c|}{256 OFDM } & \multicolumn{2}{|c|}{128 OFDMA } & \multicolumn{2}{|c|}{512 OFDMA } & \multicolumn{2}{|c|}{1,024 OFDMA } \\
\hline Oversampling & \multicolumn{2}{|c|}{$8 / 7$} & \multicolumn{2}{|c|}{$28 / 25$} & \multicolumn{2}{|c|}{$28 / 25$} & \multicolumn{2}{|c|}{$28 / 25$} \\
\hline \multirow{2}{*}{$\begin{array}{l}\text { Modulation } \\
\text { \& Code Rate }\end{array}$} & \multicolumn{8}{|c|}{ PHY-Layer Data Rate (kbps) } \\
\hline & DL & UL & DL & UL & DL & UL & DL & UL \\
\hline BPSK, $1 / 2$ & 946 & 326 & \multicolumn{6}{|c|}{ Not applicable } \\
\hline QPSK, $1 / 2$ & 1,882 & 653 & 504 & 154 & 2,520 & 653 & 5,040 & 1,344 \\
\hline QPSK, $3 / 4$ & 2,822 & 979 & 756 & 230 & 3,780 & 979 & 7,560 & 2,016 \\
\hline $16 \mathrm{QAM}, 1 / 2$ & 3,763 & 1,306 & 1,008 & 307 & 5,040 & 1,306 & 10,080 & 2,688 \\
\hline 16 QAM, 3/4 & 5,645 & 1,958 & 1,512 & 461 & 7,560 & 1,958 & 15,120 & 4,032 \\
\hline $64 \mathrm{QAM}, 1 / 2$ & 5,645 & 1,958 & 1,512 & 461 & 7,560 & 1,958 & 15,120 & 4,032 \\
\hline 64 QAM, 2/3 & 7,526 & 2,611 & 2,016 & 614 & 10,080 & 2,611 & 20,160 & 5,376 \\
\hline 64 QAM, 3/4 & 8,467 & 2,938 & 2,268 & 691 & 11,340 & 2,938 & 22,680 & 6,048 \\
\hline 64 QAM, 5/6 & 9,408 & 3,264 & 2,520 & 768 & 12,600 & 3,264 & 25,200 & 6,720 \\
\hline
\end{tabular}

4.1.4 Duplexing : PHY layers support duplexing, where a station's concurrent transmission and reception, is possible through time division duplex and frequency division duplex. In TDD, a station transmits then receives (or vice versa) but not at the same time. This option helps reduce subscriber station costs, because the radio is less complex. In FDD, a station transmits and receives simultaneously on different channels. PMP supports both TDD and FDD while Mesh mode only uses TDD duplexing [9].

\subsection{MAC LAYER}

The primary task of the WiMAX MAC layer is to provide an interface between the higher layers and the physical layer. The 802.16 MAC was first designed for point-to-multipoint (PMP) applications and is based on collision sense multiple access with collision avoidance (CSMA/CA). Several features characterize the MAC layer in WiMAX which makes it an efficient broadband technology.

\subsubsection{Main Features of MAC Layer}

The MAC layer in WiMAX has unique characteristics and features that make it suitable for almost all of the known broadband applications with different mobility rates. 
International Journal of Computer Science \& Engineering Survey (IJCSES) Vol.2, No.1, Feb 2011

- MAC layer incorporates higher security by introducing the Privacy key management. PKM that uses extensible authentication protocol.

- It has a special Multicast and broadcast support initially built as a separate multicast/broadcast zone in PMP mode.

- It introduces multiple Manageability primitives.

- It is Connection Oriented, where all service flows are mapped into a unique connection identifier CID for all services including services issued by connectionless applications at higher layers.

- It has an efficient handover and mobility management primitives through soft and hard handover mechanisms

- It allows sleep and idle power management modes in addition to normal mode to increase the life time of mobile devices.

- Mac layer also supports Header suppression and fragmentation which implies more efficiency in utilizing Total bandwidths.

- IT has five service classes to be described in more details later in this paper and they are, unsolicited grant service (UGS), real-time polling service (rtPS), non-real-time polling service (nrtPS), best effort (BE) and extended real-time variable rate (ERT-VR) service.

The MAC layer features combined with the PHY layer features like the SOFDMA makes WiMAX an appealing technology for applications that requires high data rate transmission such as VOIP and multimedia applications.

\subsubsection{MAC Frame Structure}

The MAC layer receives service requests from the upper layer that is represented as MAC service data units (MSDUs). The MAC layer then arrange them into MAC layer protocol data units (MPDUs) and send these data units to lower PHY layers and it collects the MPDUs into MSDUs when it receives them from PHY layer. MPDUs in the MAC layer could have different lengths. The WiMAX MAC layer can combine multiple variable sized MPDUs in one burst to reduce the PHY overhead. It also can combine more than small sized MSDU coming from same upper layer service into one MPDU reducing overhead at MAC layer level. In the other hand larger MSDUs can be divided to smaller MPDUs that makes MAC layer flexible and efficient. Figure 2. shows an example of various MAC PDU frames used with different data packets. Each MPDU frame contains a generic MAC header with connection identifier (CID), in addition to the length, and bits used for cyclic redundancy check (CRC), and other control data about encryption method and keys if any. The MAC payload contains communication message or a management message. The communication message contains the MSDUs and in some cases may contain bandwidth requests or retransmission requests. Automatic Repeat Request (ARQ) mechanism is also supported by WiMAX MAC which allows retransmission of MSDUs which achieves better reliability [8]. 
(a) MAC PDU frame carrying several fix ed length MSDUs packed together

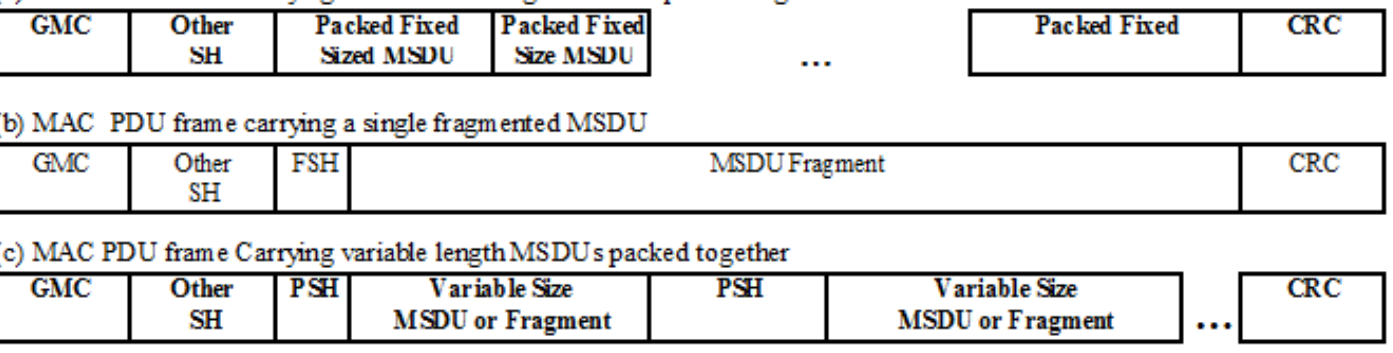

(d) MAC PDU frame carrying ARQ payload

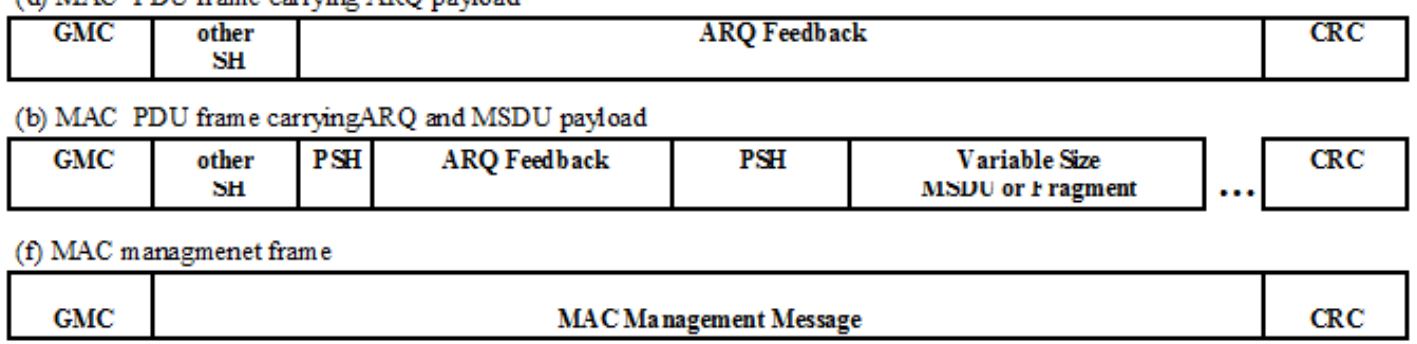

Figure 2. Examples of various MAC PDU frames where CRC is Cyclic Redundancy Check, FSH is theFragmentation Subheader, GMH is the Generic MAC Header, PSH is the Packet Subheader and the SH stands for Subheader

\subsubsection{MAC Sublayers}

The IEEE standards for WiMAX in 2004 and 2005 have defined a convergence sub layer, common part sublayers and the security sub layer as part of the MAC layer. The Convergence sub layer maps the transport layer traffic to a MAC according to the type of traffic to be scheduled and handled according to its quality of service requirement like ATM, TDM Voice, Ethernet, IP, and any other. Convergence sub layer is also responsible for MSDU header suppression that reduces upper layer overheads. Common Part Sub layer performs regular MAC layer functions; it uses TDM multiplexing on the Downlink and allows sharing the uplink between SSs in TDMA fashion. Common part sub layer also maps all services, including upper layers connectionless services, to a unique connection identifier (CID). Common part sub layer is the layer to provide, Grant/request mechanism, associates QoS parameters, and routing data to the correct convergence sub layer and provides downlink scheduling services. The MAC security sub layer is responsible for access authentication, connection setup and providing key exchange during the network entry procedure and encryption for data privacy.

\subsubsection{QoS Support in MAC Layer}

The most important part of the MAC layer design that distinguishes WiMAX from all other broadband wireless standards is its support for QoS classes. MAC architecture provides a connection oriented architecture which helps achieving a good control of QoS, where the Base station is responsible for controlling all downlink and uplink connections. A certain set of QoS parameters could be associated with a service flow which is a unidirectional flow of packets identified by a service flow identifier (SFID). WiMAX has five classes of flows, each having different QoS requirements, and these are: Unsolicited Grant Service (UGS), Real-Time Polling Service (rtPS), non-Real-Time Polling Service (nrtPS), Enhanced-Real-Time Polling Service (ertPS), and best effort (BE). Table 3. [11] shows a brief description of each service class with it 
QoS requirement. Also for each class's uplink connections, the type of service specifies which mechanisms to use in order to request bandwidth [12].

UGS support applications that require stringent delay requirements with fixed data packet on a periodic manner such as VoIP. The UGS service doesn't request bandwidth instead bandwidth for uplink is granted regardless of the channel quality estimate hence, UGS connections use the unsolicited granting bandwidth-request. A periodic bandwidth is granted for any UGS service class without any polling or contention. The bandwidth granted to each UGS service is determined by the BS as the average of lowest amount of data transmitted on the connection when over time. SS may ask the BS to poll it to additional allocated bandwidth if it needs more bandwidth.

Real-time polling service rtPS is designed to support applications with less delay requirements and has variable size data frames at periodic intervals, such as video streaming of (MPEG) video and streaming audio applications. In rtPS connection the QoS guarantees it supports are a minimum reserved bandwidth with an upper threshold for waiting times for each packet at the MAC layer and a minimum latency. Unlike the UGS, packet sizes in rtPS are of variable length and a SS is required to inform the BS of its current bandwidth at the time of service request. For each rtPS connection the BS grants unicast polls every polling interval specified by QoS parameters. nrtPS and BE are used with applications which doesn't have any strict delay requirements. nrtPS is used with application that requires a minimum data rate, like File Transfer Protocol (FTP) while BE doesn't need minimum data rate requirement as an example is HTTP and email applications . nrtPS and BE uses piggybacking to embed the bandwidth request into an uplink PDU or by replying to a broadcast poll initiated by the base Station which in a contention based basis

Table 3. QoS Classes Supported by WIMAX MAC layer

\begin{tabular}{|l|l|l|}
\hline & \multicolumn{1}{|c|}{ Description } & \multicolumn{1}{|c|}{ App lica tions } \\
\hline Unsolicited & For Constant Bit Rate & VOIP \\
Grant Service & (CBR) and delay- & \\
dependent applications & & \\
\hline Real-Time & For Variable Rate and & Streaming audio, Streaming \\
Polling & delay dependent & video \\
Service (rtPS) & applications & \\
\hline Extended & For Variable Rate and & VOIP with silence \\
Real-Time & delay dependent & suppression \\
Polling & applications & \\
Service (ertPS) & & \\
\hline Non-real-time & Variable rate and non- & FTP \\
Polling & real time applications & \\
Service & & E-mail, web traffic \\
(nrtPS) & & \\
\hline Best Effort & Best Effort & \\
(BE) & & \\
\hline
\end{tabular}


International Journal of Computer Science \& Engineering Survey (IJCSES) Vol.2, No.1, Feb 2011

\subsubsection{Network Entry Procedure and Grant Mechanisms in WiMAX}

- PMP mode: When a subscriber station wishes to register itself with a BS in a PMP deployment the first thing the subscriber station does is to tune up with a downlink channel (DL) and an uplink channel (UL) to get the frame structure of the UL which is called ULMAP from the BS. Then the Ranging Procedure starts by aligning the subscriber station for transmission to the correct mini slot boundary specified by the base stations. Subscriber station establishes IP connectivity with the Base stations and as a final step for completing registration base station exchanges time and security parameters with the Subscriber stations. After successful registration stations request for transmission opportunities on the uplink UL channel. Base station gathers these requests and determines the grant size and number of time slots that each SS will be allowed to transmit in the UL Frame. This information is broadcasted in the downlink (DL) channel by the BS using the UL-MAP message at the beginning of each DL-Frame, The UL-MAP contains Information Elements (IE) that describes the transmission opportunities in the UL channel, such as initial maintenance, station maintenance, contention, and reservation access. A SS receiving ULMAP will transmit data in the predefined transmission opportunities indicated by IE. Transmission opportunities are assigned by the BS using QoS agreements to support a certain service class requested by SS's.

- Mesh mode: Network entry procedure for mesh modes differs totally from that for PMP mode. In mesh Mode current nodes connected to the mesh network periodically broadcast Mesh Network Configuration messages. These messages provide information about the current configuration of the Network through a field called Network Descriptor. A node wishing to join this mesh listens first to any Mesh Network Configuration message on its range. The new node selects closest neighbor as its host node and uses it to send its Mesh Network Entry request with registration information to the BS. The Mesh BS registers the new node and adds it as the child node of the host node and broadcasts an update configuration messages to all the nodes in the network [18]. Unlike PMP mode the WiMAX forum didn't identify the request and grant mechanisms for this mode. All communication is done through Distributed or Centralized scheduling with exchange of scheduling messages $\mathrm{MSH}-\mathrm{DSCH}$ and MSH-CSCH described later in this paper.

\section{SCHEDULING IN WiMAX}

Although IEEE 802.16 standards specify several QoS schemes and related message formats, the problem of scheduling algorithms for both PMP and Mesh mode are left unsolved and left as an open research issue.

\subsection{Scheduling in PMP mode [12][14][15]}

In PMP mode, the BS is responsible for a scheduling decision for SS. Each frame is assigned a subcarrier in a certain time slot with scheduling decision made on a frame by frame basis. The time period and the sub channel assigned to frames is done in scheduling decision for each SS by BS and broadcasted to all users in multicast group if any or sent to the user with unicast request. A scheduling decision in WiMAX should satisfy the QoS requirements for each requesting service. Scheduler to be defined in this mode is a downlink and uplink scheduler for the base station and only an uplink scheduler for the subscriber station. In [9] and [11] the scheduling algorithms for MAC layer were categorized into two main categories, channel unaware and channel aware scheduling algorithms. Channel unaware are further divided into homogeneous and hybrid algorithms. Channel aware are known also as opportunistic algorithms.

Homogeneous Schedulers: These are referred to the assigning one classical scheduling algorithm for all classes of service not taken into consideration the varying condition of 
International Journal of Computer Science \& Engineering Survey (IJCSES) Vol.2, No.1, Feb 2011

subscriber channel. Examples of such algorithms are the round robin, deficit round robin, weighted round robin, and earliest deadline first. None of the homogeneous algorithms provided the required fairness and QoS guarantees for all the services classes introduced in WiMAX. For example if earliest deadline first suits the QoS requirement of rtPs service class, it will work poorly with UGS.

Hybrid Schedulers: The Second category algorithms were introduced to overcome the problem incurred by using one homogeneous scheduling algorithm. Some algorithm that uses a hybrid approach uses different scheduling strategies for each service class. One uses earliest deadline first (EDF) for rtPS service class, uses Weighted Fair Queue (WFQ) for nrtPS and a First In First Out Scheduling algorithm for the Best Effort Service class. In this hybrid algorithm bandwidth for all classes is allocated using priority to allow fairness between all types of the service classes. .Another Hyprid scheme uses combinations of EDF and WFQ with fair bandwidth sharing between nrtPS and BE cthat both assigned WFQ, the authors did not describe the mechanism for fair allocations. $\operatorname{In}[31]$ the authors proposes a hybrid scheduling algorithm that assigns bandwidths or transmission opportunities based on a priority mechanism with weighted round robin nrtPS and rtPS and round robin scheduling method for other classes. At first opportunities are assigned to nrtPS and rtPS.s until their QoS guarantees are fulfilled. BE requests from other SS are then served using with any remaining bandwidth using the round robin approach. Hybrid schemas provided some fairness between the different qualities of service classes but again didn't take into consideration the variable channel conditions of each subscriber station and the flexibility of WiMAX by providing different bandwidth grant size for different quality of service classes. Some studies proposed other mechanisms that should be used with scheduling algorithms such an admission control procedure and a traffic policing mechanism.

Channel Aware / Opportunistic Scheduling: Opportunistic Scheduling is the kind of algorithms designed specifically for WiMAX to achieve the best quality of services for various services classes within a variable channel condition. Such algorithms where also called cross layer since their scheduling decision is based on channel condition estimation provided by the PHY layer to the MAC layer. A Temporary Removal (TR) scheduler in [28] is an example of opportunistic algorithms in which the scheduler uses information from lower level to identify service packets power for each SS. Packets with low power are temporary removed from the scheduling queue for a time specified by a TR timer. Only SS with packets that can be served is left on the queue. SS that were removed from the queue are checked again after timer expires and is returned to the queue if its packet power increases. For Each packet there is a limit for number of times a packet is checked for better channel condition quality and is unconditionally added to top of the queue. In [29] an Opportunistic Deficit Round Robin scheduler (O-DRR) A BS uses periodic polls to identify the SS to be served. The decision to include any of the polled SSs in the scheduled set is built upon the channel conditions and radio quality of each one. Any SS to be given an opportunity to send during the scheduling frame should have data to be transmitted and should have SIR above a certain threshold value. Both TR and O-DRR didn't consider the different service classes and made its scheduling decision based on the channel quality only. In [30] Frame Registry Tree Scheduler (FRTS) the authors used a deadline criterion for the scheduler to assign time frames for SS packets. .The Deadline is the same as arrival and latency time For UGS and rtPS services, while for nrtPS and BE services there is no packet deadline specified. Higher priorities are given to UGS and rtPS services over other classes. Scheduling decision will be evaluated one any SS changes its modulation type or QoS requirement. Some Opportunistic Scheduling algorithms was proposed to deal with only one type of Services Classes such as The adaptive rtPS scheduler in [30]. This algorithm tried to reduce the delay incurred between multiple grant/request operation between the SS and the BS for multiple packets arriving at different times. The SS here will be assigned time slots for the packets currently in the SS queue and for other packets that is expected to arrive from upper 
layers. The basic idea of the adaptive rtPS scheduler is to propose an rtPS bandwidth request process in which the subscriber requests time slots for the data present in the rtPS queue and also for the data which will arrive. Authors have defined a prediction method to estimate packet arrival times. In [31] authors introduced Cross-Layer scheduling algorithm in which Scheduling opportunities and time slots are assigned based on a priority. Priority for each SS is given according to its channel condition and its service request class.

\subsection{Scheduling for Mesh Mode [11][13]}

Most researches for scheduling and routing done for wireless mesh network were based on the IEEE 802.11 standard. These researches could be used with WiMAX mesh, but will not produce efficient results since it wasn't build based on the characteristics of WiMAX technology and there was only few researches done specifically for WiMAX mesh networks. Mesh mode at the PHY layer supports only Time Division Duplexing (TDD) and hence at the MAC layer the subscriber stations compete for transmission opportunities using TDMA (Time Division Multiple Access). A frame in WiMAX mesh frame consists of two main sub frames, one dedicated for carrying control information and the other is for carrying data. The Control sub frame carries information about the connections establishment/maintenance and scheduling of data transmission between different SSs.

Only some nodes in the mesh network can be used to connect the mesh network to the backhaul links in the same fashion as BS do. Scheduling in mesh mode is built over scheduling trees that is rooted at the BS. Mesh can use centralized scheduling or distributed Scheduling schemas. When using Mesh centralized scheduling, the BS nodes perform much of the same basic functions as do the BS in PMP mode. Thus, the key difference is that in Mesh mode all the SSs may communicate directly with other SSs eliminating the need for direct connections between SSs and BS of the Mesh network. In Centralized Scheduling Algorithms Communication between all Stations is controlled by a centralized algorithm provided by the BS. In distributed Scheduling direct communication or links can be coordinated by all nodes periodically.

\subsubsection{Distributed Scheduling}

In Distributed Scheduling SS's stations may contribute in the scheduling decisions and hence each node in the mesh network is aware of its neighbours. A neighbor is the node with direct connection or is separated by multiple node. Distributed scheduling is classified as either coordinated or uncoordinated. Subscriber nodes in addition to the base stations coordinate's their communications with all other nodes in a two hop neighbourhood. Nodes Shares the scheduling information through the control part of each frame it transmits. Neighbour stations use the same channel for exchanging scheduling. Scheduling decision in coordinated distributed scheduling doesn't rely on the BS and can be directed to any other node in the neighbourhood. In the other hand Uncoordinated distributed scheduling is based on direct request and grant mechanism between two nodes. Uncoordinated is suitable most for schedules that is required to be fast. Uncoordinated distributed schedules are established by a direct requests and grants between two communicating nodes. In uncoordinated scheduling competing for the shared radio channel is not governed by neighbourhood status as in coordinated, and may collide with other scheduling messages from either coordinated or centralized scheduling. Distributed Scheduling is done by the exchange of MSH-DSCH messages.

\subsubsection{Centralized Scheduling}

Centralized Scheduling refers to algorithms for mesh networks where communication and data transmission taken place between BS and other nodes in the Mesh. In Centralized Scheduling the Mesh Centralized Scheduling Configuration (MSH-CSCF) message is broadcasted by Bs to inform all nodes of the current configuration and routing tree data of the mesh, each node then 
International Journal of Computer Science \& Engineering Survey (IJCSES) Vol.2, No.1, Feb 2011

forwards this message to its neighbours. The Bs then BS gathers resource request from SSs using Mesh Centralized Scheduling (MSH-CSCH) messages. Flow schedule is assigned to the requesting SS and broadcasted. SS Then uses the flow assignment to determine its actual transmission opportunities using a predetermined algorithm. In this type a BS plays the same role as in PMP mode but with no direct connection between BS and SSs. SS will also use MSH$\mathrm{CSCH}$ messages to report its request change to the BS. BS in response adjusts its flow assignment and broadcast it again to all nodes. Frames scheduled to be transmitted in one MSH- CSCH message is governed by how many frames are needed for the following Schedule to be packed and distributed. The MSH-CSCH message carries data about the transmission period for each node, the last node to receive the schedule and the time taken by the BS to send the current schedule. The process of broadcasting the centralized messages starts at the BS down the tree and the hop count is incremented until all nodes receives the grant or the configuration message. Gathering the request messages starts from the farthest node in the tree with the largest hop count and propagates up the tree until it reaches the BS.

\subsubsection{Algorithms for WiMAX mesh}

Centralized and distributed scheduling is two approaches used in general with any networks with mesh topology and is also applicable to WiMAX mesh. Studies that proposed scheduling schemas specifically for WiMAX mesh was also conducted. In [21] a Scheduling schema was proposed in which the authors tried to exploit more of the capabilities of the WiMAX technology for a better throughput. They make use of parallel transmission for this purpose. For each scheduling round, the number of active connections is determined by the scheduler through exchange of scheduling messages. The connection that has more traffic requests is allocated the next traffic opportunity. In this schema links that has high interference is excluded. This process is repeated until there is no unallocated traffic.

In [11], a centralized scheduling and routing tree construction algorithms was introduced. This algorithms schedules each service flow individually to serve better each flow QoS requirements. Even if this algorithms serves better the QoS guarantees for each service flow but it assumes only one transmission link is active per time slot in the whole mesh network which is not practical and will reduce the throughput of the network. An efficient scheduling schema should exploit the capability of concurrent transmission on non interfering mesh network links.

A fair scheduling schema for WiMAX was proposed in In [16]. In this paper the authors followed a fairness model for the assignment of scheduling opportunities for all traffic requests of all types. They formulated a problem to get the best throughput while making scheduling decisions that satisfies the fairness criteria. There algorithm was based on finding an optimal fair schedule obtained by optimizing the formulated problem.

\section{Multicasting Approaches in WiMAX}

Multicasting is an efficient and crucial scheme to be used in all broadband technologies to reduce the bandwidth cost for applications that sends the same data to multiple recipients. Multicasting in PMP mode takes a much simpler way than multicasting in Mesh mode. In PMP mode the BS MAC layer has defined a special multicast broadcast service (MBS) while in Mesh mode multicasting is build over routing algorithms introduced for Mesh networks.

\subsection{Multicasting PMP mode}

The WiMAX Multicast Broadcast Service (MBS) was defined for PMP mode specifically for broadcasting or multicasting data or video over the WiMAX air interface. The MBS zone is built using either one BS or multiple BSs in the same region such that all sends the same broadcast or multicast message at the same rate at the same frequency channel. For each MBS 
service a down link frame can be assigned totally for the MBS messages or can use a separate MBS zone in the down link frame. BSs in this service should maintain time synchronization when sending broadcast/multicast messages, use one connection identifier CID and use same security association (SA) information for encryption of broadcast/multicast messages. Each BS can be part of multiple MBS zones and each zone can have multiple BSs. SSs gets information about MBS zone from The BS transmitted data. The SSs uses the Downlink map to confirm the MBS zone. At the same time, there is an MBS-MAP in an MBS zone, which contains information about the location of current MBS packets and the time when the next MBS packet is transmitted.

A connection Id is assigned for all services at the Mac layer and for the MBS service a Multicast connection ID (MCID) is assigned for each packet. MBS is defined for PMP and hence it can use either TDD or FDD modes. MBS features make it an efficient way to multicast/broadcast streaming data to multiple users using a shared radio channel.

Using MBS service at MAC layer for Multicasting implies that one MCID is assigned for the service flow to satisfy the QoS requirements and will be assigned one channel with a specific modulation and coding schemas. The problem arises when the users in multicast group has the same quality of service requirement but has different channel conditions, so which channel and at what modulation rate should the Multicast service be assigned. Several multicasting schemas where proposed for handling this issue.

A Cooperative Multicasting Schemas in [17] tried to reduce the differences in channel conditions between SSs in the same multicast group. Such Schemas has an assumption that SS's can communicate directly and can relay data to each other. In such schemas the BS sends multicast data at a high rate for users with very good channel condition. User getting this multicast cast can relay it to other users with bad channel conditions. To control this relay operation and making each SS knows what data it gets and what data to relay, authors combined this idea with concept of random network coding. A Cooperative Multicasting with network coding then works well with the new WiMAX standard 802.16j where PMP mode is extended to MMR mode and where nodes can works as relays.

In another multicasting approach [18], the multicast users in the multicast group are divided into two sub-groups. Users in each group are selected based on their channel conditions. To better adjust to the quality of channel conditions for each group two copies of multicast data are sent in different time slots. Each copy is modulated and coded with different values with two different data rates. This schema was shown to enhance the overall throughput. However, it has limitations and will not be very efficient when the number of users in the poor condition group is very small.

In [19], authors didn't make any effort to calculate optimal rate for all multicast users and instead they focused on finding an optimal rate for a certain subset of multicast group for each transmission opportunity. They showed that it works well on the one hop shared channel scenario, but it does not consider the cooperative diversity in the broadcasting channels.

Other research on multicasting for real time video in [20] makes use of video layering techniques to divide video into multiple layers and send these layers through a multicast channel where user with good channel condition can receive all the video layers at the full multicasting rate and users with poor channels will watch a low quality video because they can receive only the basic layer of the video.

\subsection{Multicasting Mesh Mode.}

The WiMAX standard defined the MBS service for PMP mode in a single hop route. In mesh mode a mesh network is managed by a mesh base station that provides an interface to external networks and acts as the central control node to the mesh network. Multicasting in mesh modes 
International Journal of Computer Science \& Engineering Survey (IJCSES) Vol.2, No.1, Feb 2011

depends on routing approaches which is built over scheduling trees. Scheduling trees are built depending on the actual location of SSs and are rooted at the BS. As any other broadband technology, multicasting schemas for WiMAX mesh networks follow the multicasting approaches and researches done for wireless ad hoc networks. These protocol runs on networks with same topology as WiMAX, and called tree-based protocols as the Multicast operation od Ad hoc On demand Vector (MAODV) protocol [24], and the adaptive Demand Driven Multicast Routing (ADMR) [25]. In Both protocols a sender can have one path until for a predefined receiver. Tree based multicast protocols could be either source-tree-based or sharetree-based. In source-tree-based, the tree is rooted at the source and at each multicasting session a new multicast tree is created multicast tree. In shared-tree multicast protocols, a shared multicast tree is built once and is not necessarily rooted at the source. Some protocols for Ad hoc networks tried to reduce the overhead and delay produced by frequent topology change due to nodes joining and leaving the network or moving within the same network.

Few researches in literatures addressed multicasting in WiMAX mesh mode. All researches done are for wireless mesh networks based on 802.11 families of standards. One study for WiMAX in [22] proposes a multicast protocol based on building a high source efficient multicast tree that uses messages defined for centralized and distributed scheduling in the WiMAX standard. They introduce an easy to implement tree construction mechanism based on the adjustment of the centralized routing tree. In [11], authors studied a cross layer routing and centralized scheduling schema. They showed that a good performance can be obtained if combining the shortest path routing algorithm with a scheduling algorithm that works on a per time slot maximizing criteria as discussed in the scheduling part of this paper. Another joint schema in [32] proposed for TDMA based mesh networks in general. In this work an integrated QoS routing and scheduling schema was proposed. In this schema QoS guarantees for TDMA mesh networks was shown to be fulfilled and the authors formulated a linear programming optimization models to find the non collision bandwidth in a specific path.

\section{Conclusions}

In this paper we conducted a broad study of WiMAX. We started by describing main features and the evolution of the standards with focus on the current working standards IEEE 802.16d \& e. Like all 802.x families, the standards define only the MAC and the PHY layers to allow interoperability with higher layers and other standards. PHY layer uses modulation and coding schemas combined with OFDMA to produce variable data rates to support variable channel conditions. MAC layer in WiMAX has many unique features from which supporting different quality of service classes is the most important. By giving an overview of both MAC and physical layers and differentiate between the two modes of operations helps understands the research issues in terms of scheduling and Multicasting. WiMAX provided support for QoS classes but didn't define the scheduling mechanism for those classes in all modes. In this survey we addressed and compared different scheduling approaches defined for WiMAX PMP and Mesh modes. We also studied the multicasting schemas in PMP modes which is based on the Multicast Broadcast service already defined in MAC layer for PMP mode, and showed how multicasting schemas in Mesh mode follows the multicasting schema used for wireless mesh networks defined for IEEE 802.11. It is worth to mention that the current working standard approved in late 2009 is the $802.16 \mathrm{j}$ that supports MMR mode of operation, and withdraw the Mesh Mode. MMR mode has a tree structure which is a special structure a mesh can follow, so part of researches and schemas used for mesh mode can be alleviated to work with MMR mode. This study gives a good reference or a good start for understanding WiMAX technology and the main research issues. 
International Journal of Computer Science \& Engineering Survey (IJCSES) Vol.2, No.1, Feb 2011

\section{REFERENCES}

[1] "WiMAX End-to-End Network Systems Architecture - Stage 2: Architecture Tenets, Reference Model and Reference Points," WiMAX Forum, December, 2005.

[2] Kejie Lu, Yi Qian, and Hsiao-Hwa Chen, "A Secure and Service-Oriented Network Control Framework for WiMAX Networks"," IEEE Communications Magazine, Vol. 45, No. 5, pp. 124130, May 2007.

[3] M. Lee et al., "Emerging Standards for Wireless Mesh Technology," IEEE Wireless Commun., vol. 13, no. 2, Apr. 2006, pp. 56-63.

[4] IEEE 802.16-2004, "IEEE standard for Local and Metropolitan Area Networks - Part 16: Air Interface for Fixed Broadband Wireless Access Systems," Oct. 2004.

[5] IEEE Std 802.16e-2005, "IEEE Standard for Local and metropolitan area networks--Part 16: Air Interface for Fixed Broadband Wireless Access Systems--Amendment 2: Physical and Medium Access Control Layers for Combined Fixed and Mobile Operation in Licensed Bands," Feb. 2006.

[6] IEEE 802.16 Working Group 16: IEEE Standard for Metropolitan Area Networks, Part 16: Air Interface for Fixed and Mobile Broadband Wireless Systems - Multihop Relay Specification. IEEE 802.16's Relay Task Group 2008.

[7] C. Nie, T. Korakis, S. Panwar, "A Multi-hop Polling Service with Bandwidth Request Aggregation in IEEE 802.16j Networks", Proceedings of IEEE VTC 2008, Marina Bay, Singapore, May 2008.

[8] Abichar, Z.; Yanlin Peng; Chang, J.M.; WiMax: The Emergence of Wireless Broadband. IEEE journal, Volume 8, Issue 4, July-Aug. 2006 Page(s):44 - 48.

[9] Fourty, N.; Val, T.; Wei, A.; A WiMAX Physical Layer Use for Emergency Audio Communications. WIMOB 2009. IEEE International Conference on 12-14 Oct. 2009 Page(s):435 440 .

[10] Loutfi Nuaymi, WiMAX: Technology for Broadband Wireless Access. John Wiley and Sons, Chichester, England, 2007

[11] H. Shetiya and V. Sharma. Algorithms for routing and centralized scheduling to provide QoS in IEEE 802.16 mesh networks. ACM workshop on Wireless Multimedia Networking and Performance Modelling, October 2005.

[12] Aymen Belghith and Loutfi Nuaymi, "Comparison of WiMAX scheduling algorithms and proposals for the rtPS QoS class", 14th European Wireless 2008, EW2008, pages 1-6, Prague, Czech Republic, 22-25 June

[13] H. Wei, S. Ganguly, R. Izmailov. Interference-Aware IEEE 802.16 WiMax Mesh Networks. in Proceedings of 61 st IEEE Vehicular Technology Conference (VTC 2005 Spring), Stockholm, Sweden, May 29-June 1, 2005.

[14] C. So-In, R. Jain, A. Tamimi. MAC layer Mechanism for Wireless WiMAX Networks with Mesh Topology. IEEE JOURNAL ON SELECTED AREAS IN COMMUNICATIONS, VOL. 27, NO. 2, FEBRUARY 2009

[15] Yong Zhang; Anfeng Mao; Guo Ping; Ning Hong; Xu Guang; Analysis of Quality of Service (QoS) in WiMAX networks Pervasive Computing and Applications. ICPCA 2008. Third International Conference Volume 2, 6-8 Oct. 2008 Page(s):882 - 886.

[16] M. Cao, V. Raghunathan, and P. R. Kumar., "A tractable algorithm for fair and efficient uplink scheduling of multi-hop wimax mesh networks", In Proceedings of 2nd IEEE Workshop on Wireless Mesh Networks (WiMesh 2006), September 2006

[17] Jin Jin, Baochun Li. "Cooperative Multicast Scheduling with Random Network Coding in WiMAX," in the 17th IEEE International Workshop on Quality of Service (IWQoS 2009), Charleston, South Carolina, July 13-15, 2009 
International Journal of Computer Science \& Engineering Survey (IJCSES) Vol.2, No.1, Feb 2011

[18] P. K. Gopala and H. E. Gamal, "On the Throughput-delay Tradeoff in Cellular Multicast," in Proc. of International Conference on WirelessNetworks, Communications and Mobile Computing, June 2005 .

[19] U. C. Kozat, "On the Throughput Capacity of Opportunistic Multicasting with Erasure Codes," in Proc. of IEEE INFOCOM, 2008.

[20] S. Deb, S Jaiswal, K. Nagaraj, Real-Time Video Multicast in WiMAX Networks. In Proc of IEEE INFOCOM 2008.

[21] Chen J, Jiao W, Jiang P, Guo Q, A multicast mechanism in WiMax mesh network. In: Asia-Pacific conference on communication (APCC 2006), pp 1-5, August 2006.

[22] F. Xie, K. Hua , N. Jiang. Achieving True Video-on-Demand Service in Multi- Hop WiMax Mesh Networks. 32nd IEEE Conference on Local Computer Networks 2007.

[23] Ian F. Akyildiz, Xudong Wang, Weilin Wang, "Wireless mesh networks: a survey," Computer Networks Journal (Elsevier), January, 2005

[24] E. M. Royer, C. E. Perkins. "Multicast Operation of the Ad-hoc On Demand Distance Vector Routing Protocol", Proceeding of the fifth annual ACMNIEEE international conference on mobile computing and networking, pp. 207-218,1999.

[25] C. Siva Ram Murthy, B.S. Manoj, "Ad Hoc Wireless Networks: Architectures and Protocols", Prentice Hall, Hardcover, Published May 2004

[26] H. Wei, S. Ganguly, A. Izmailov, and Z. Haas, "Interference-Aware IEEE 802.16 WiMax Networks", in Vehicular Technology Conference, 2005. VTC 2005-Spring. 2005 IEEE 61st, Vol. 5; 3102-3106.

[27] W. Lilei and X. Huimin, "A new management strategy of service flow in IEEE 802.16 systems," in Proc. IEEE Conf. Industrial Electronics and Applications., Harbin, China, 2008, pp 1716-1719.

[28] C.F. Ball, F. Treml, X. Gaube, and A. Klein, "Performance Analysis of Temporary Removal Scheduling applied to mobile WiMAX Scenarios in Tight Frequency Reuse", the 16th Annual IEEE International Symposium on Personal Indoor and Mobile Radio Communications, IMRC'2005,Berlin, 11 - 14 September 2005.

[29] H. K. Rath, A. Bhorkar, and V. Sharma, "An Opportunistic DRR (O-DRR) Uplink Scheduling Scheme for IEEE 802.16-based Broadband Wireless Networks", IETE, International Conference on Next Generation Networks (ICNGN), Mumbai, 9 February 2006Chania, Greece, 18 - 21 September 2005.

[30] R. Mukul, P. Singh, D. Jayaram, D. Das, N. Sreenivasulu, K. Vinay, and A. Ramamoorthly, "An Adaptive Bandwidth Request Mechanism for QoS Enhancement in WiMax Real Time Communication", Wireless and Optical Communications Networks, 2006 IFIP International Conference on, Bangalore, India, 11 - 13 April 2006.

[31] M.Settembre, M.Puleri, S.Garritano, P.Testa, R.Albanese, M.Mancini and V.Lo Curto "Performance analysis of an efficient packet-based IEEE 802.16 MAC supporting adaptive modulation and coding”, International Symposium on Computer Networks, pp.11-16, June 2006.

[32] Hong C-Y, Pang A-C, Wu J-LC (2007) QoS routing and scheduling in TDMA based wireless mesh backhaul networks. In: IEEE wireless communications and networkingconference (WCNC 2007), March 


\section{Authors}

Manal AL-Bzoor is a Ph.D. student in Computer Science and Engineering at the University of Connecticut. She has received her B.S. degree from Jordan University of Science and Technology -Jordan and her MS degree in Computer Engineering from University of Michigan-Dearborn. She worked as an Instructor at Computer Engineering Department - Yarmouk University Jordan (2006-2009) where she supervised multiple projects in wireless networks and in image processing. Manal current research interests are in wireless sensor networks and distributed/parallel systems.

Prof. Khaled Elleithy is the Associate Dean for Graduate Studies in the School of Engineering at the University of Bridgeport. His research interests are in the areas of network security, mobile communications, and formal approaches for design and verification. $\mathrm{He}$ has published more than one hundred twenty research papers in international journals and conferences in his areas of expertise. Dr. Elleithy is the co-chair of the International Joint Conferences on Computer, Information, and Systems Sciences, and Engineering (CISSE). He is also the editor or co-editor of 10 books published by Springer for advances on Innovations and Advanced Techniques in Systems, Computing Sciences and Software. Dr. Elleithy received his B.Sc. degree in computer science and automatic control from Alexandria University in 1983, his MS Degree in computer networks from the same university in 1986, and an MS and Ph.D. degrees in computer science from The Center for Advanced Computer Studies in the University of Louisiana at Lafayette in 1988 and 1990, respectively.
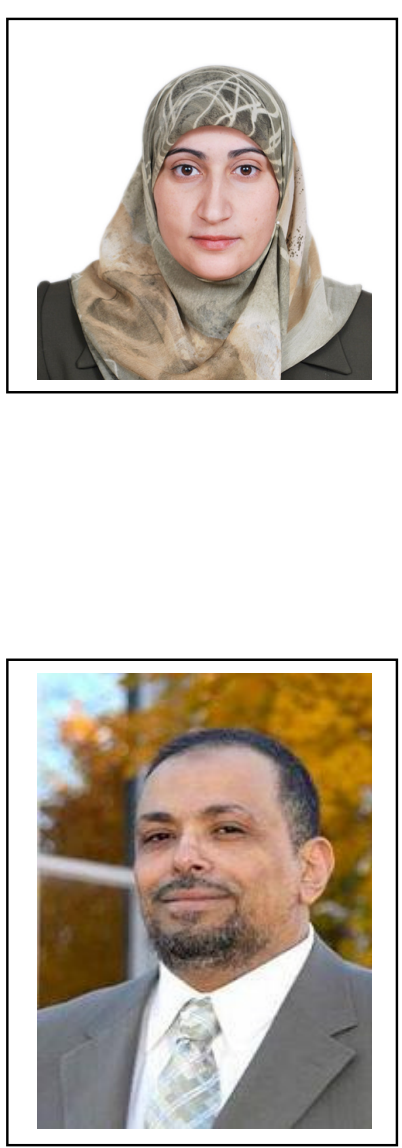